\title{
Removal of the CPAP Therapy Device During Sleep and Its Association With Body Position Changes and Oxygen Desaturations
}

\author{
Yasuhiro Yamaguchi MD PhD, Shinichiro Hibi MD PhD, Masaki Ishii MD PhD, \\ Yoko Hanaoka MD PhD, Hiroshi Yamamoto MD PhD, \\ Yasuyoshi Ouchi MD PhD, and Masahiro Akishita MD PhD
}

\begin{abstract}
BACKGROUND: Some patients with obstructive sleep apnea syndrome remove the CPAP device during sleep, although they start CPAP at bedtime. We hypothesized that body position changes and oxygen desaturations may be associated with patient removal of the CPAP device. METHODS: We consecutively enrolled 36 poor CPAP adherers and 25 good CPAP adherers. Body positions and oxygen desaturations were evaluated when these subjects used CPAP during sleep for 2 nights, permitting the removal of the CPAP device during the recording. RESULTS: Twelve poor CPAP adherers exhibited subject removal of the CPAP device within $4 \mathrm{~h}$ from the start of body position recording. The frequency of body position changes was significantly higher within $15 \mathrm{~min}$ before the CPAP removal than during other periods when CPAP was used. At the same time, oxygen desaturations were significantly more frequent within $15 \mathrm{~min}$ before CPAP removal than during other periods when CPAP was used. In addition, the majority (66.7\%) of the CPAP removal events were followed by a change from a supine position to a non-supine position within $5 \mathrm{~min}$. The number of body position changes during the first 4 -h recording was significantly higher in the poor CPAP adherers who had removed their CPAP device compared with the poor CPAP adherers who had not removed their device. However, the number of body position changes or oxygen desaturation index under CPAP during the first 4-h recording was not significantly different between the poor CPAP adherers and good CPAP adherers. CONCLUSIONS: Our findings are the first to indicate associations among CPAP removal, body position changes, and oxygen desaturations during sleep in poor CPAP adherers. However, our findings also indicated that most good CPAP adherers make frequent body position changes without removal of CPAP device, suggesting that patients can adapt to the discomfort of CPAP therapy at the time of body position changes. Key words: obstructive sleep apnea syndrome; positive airway pressure; adherence; body position changes; supine; non-supine. [Respir Care 2015;60(5):658-665. (C) 2015 Daedalus Enterprises]
\end{abstract}

\section{Introduction}

Obstructive sleep apnea syndrome (OSAS) is an important syndrome that is associated with daytime sleepiness

The authors are affiliated with the Department of Geriatric Medicine, Graduate School of Medicine, University of Tokyo, Bunkyo-ku, Tokyo, Japan.

This work was supported by grants-in-aid for scientific research from the Ministry of Education, Science, Sports and Culture of Japan and by research grants from the Mitsui Sumitomo Insurance Welfare Foundation. The authors have disclosed no other conflicts of interest. and an increased incidence of cardiovascular ${ }^{1}$ and other diseases. ${ }^{2}$ CPAP therapy is an effective treatment that reduces sleepiness ${ }^{3}$ and prolongs survival. ${ }^{4,5}$ However, poor

\footnotetext{
Correspondence: Yasuhiro Yamaguchi MD PhD, Department of Geriatric Medicine, Graduate School of Medicine, University of Tokyo, 7-3-1 Hongo, Bunkyo-ku, Tokyo 113-8655, Japan. E-mail: yamayasu-tky@ umin.ac.jp.
}

DOI: $10.4187 /$ respcare.03520 
adherence to prescribed CPAP therapy is a continuing problem in the treatment of OSAS. ${ }^{6}$

Although many studies have been conducted to evaluate the factors affecting CPAP adherence, ${ }^{7}$ the reasons for patient removal of CPAP device during sleep have not been thoroughly evaluated. Some poorly adherent patients do not remember when they removed the CPAP device during sleep, although they started CPAP therapy at bedtime. Therefore, the factors contributing to the removal of the CPAP device during sleep and the factors contributing to the lack of use of CPAP at bedtime might be different. Although education and social support might enhance the adherence to CPAP use at bedtime, ${ }^{8-11}$ other sleep-related factors might affect the removal of CPAP therapy during sleep, and may require other types of intervention.

Because some patients complain of discomfort when changing body positions or being asleep in the lateral position while using CPAP, we evaluated body positions and oxygen desaturations when subjects used CPAP during sleep and their association with the incidence of CPAP removal during sleep.

\section{Methods}

\section{Subjects}

Patients with OSAS were consecutively recruited depending on the date when they visited the hospital, because patients using CPAP therapy are required to visit a hospital at least once a month in compliance with the Japanese medical insurance system. We recruited patients with OSAS who had been treated with CPAP for $>6$ months at the University of Tokyo Hospital, and who had CPAP compliance data available for $>20$ follow-up days from September to December in the most recent 2 y. Patients with cognitive dysfunction who had difficulty in performing body position recordings at home were excluded.

Among the 89 recruited subjects, we found 41 poor CPAP adherers, who had used their CPAP device for $>4$ $\mathrm{h}$ on $<70 \%$ of the days on which they used the device. For comparison, we also consecutively enrolled 28 good CPAP adherers, who had used their CPAP device for $>4$ $\mathrm{h}$ on $\geq 70 \%$ of the days on which they used the device.

The study was performed at the Department of Geriatric Medicine, Graduate School of Medicine, University of Tokyo, Tokyo, Japan, and the study protocol was approved by the institutional review board.

\section{Diagnosis of OSAS and Prescription of CPAP Therapy}

All of the subjects were diagnosed with OSAS based on the findings of full-night polysomnography and/or full-

\section{QUICK LOOK}

\section{Current knowledge}

Obstructive sleep apnea syndrome (OSAS) is associated with daytime sleepiness and an increased incidence of cardiovascular and other comorbidities. CPAP therapy is an effective treatment that reduces sleepiness and prolongs survival. Poor adherence to prescribed CPAP therapy is commonly reported, negating the potential positive impact of therapy. The reasons for patient removal of CPAP device during sleep have not been thoroughly evaluated.

\section{What this paper contributes to our knowledge}

Frequent oxygen desaturations were seen just before CPAP removal, suggesting that suboptimal OSAS treatment would induce body position changes and CPAP removal. CPAP removal, body position changes, and oxygen desaturations during sleep were higher in subjects exhibiting poor CPAP adherence. Good CPAP adherence was associated with frequent body position changes without removal of the CPAP device, suggesting that patients can adapt to the discomfort of CPAP therapy during body position changes.

night portable polysomnography. In some subjects, fixed pressure CPAP was prescribed after confirming an adequate reduction in the apnea-hypopnea index under CPAP using polysomnography. In the other subjects, auto-mode CPAP was prescribed, and an adequate reduction in apneahypopnea index was confirmed using the records obtained from the CPAP device. All subjects using CPAP were required to visit a hospital at least once a month, and the physicians encouraged and advised the subjects to be adherent to CPAP therapy.

\section{Recording of Body Position and Arterial Oxygen Saturation During Sleep}

We recorded arterial oxygen saturation and body position using a portable sleep apnea monitoring device (Apnomonitor mini, Chest, Tokyo, Japan) at home. The recordings began automatically 15 min after turning on this device. The data for time and body position recorded by this device were validated after the recording. We advised the subjects to use their CPAP device as usual, permitting removal of the CPAP device during the recording. The subjects were blinded to the objectives of the study, that is, the recording of body position at the time of CPAP removal. We also instructed the subjects to keep a sleep 
Table 1. Baseline Characteristics

\begin{tabular}{|c|c|c|c|}
\hline & $\begin{array}{l}\text { Poor CPAP Adherers } \\
\quad(n=36)\end{array}$ & $\begin{array}{c}\text { Good CPAP Adherers } \\
(n=25)\end{array}$ & $P$ \\
\hline Age (y) & $60.6 \pm 10.0$ & $65.6 \pm 9.3$ & .06 \\
\hline Males/females $(n)$ & $29 / 7$ & $21 / 4$ & .73 \\
\hline Body mass index $\left(\mathrm{kg} / \mathrm{m}^{2}\right)$ & $28.5 \pm 5.2$ & $27.9 \pm 5.0$ & .51 \\
\hline Time (mo) & $40.8 \pm 25.0$ & $43.8 \pm 29.0$ & .79 \\
\hline \multicolumn{4}{|l|}{ After the diagnosis of OSAS } \\
\hline AHI original & $43.4 \pm 17.1$ & $35.3 \pm 16.1$ & .08 \\
\hline Auto-CPAP/fixed CPAP & $33 / 3$ & $21 / 4$ & .43 \\
\hline AHI on CPAP & $5.9 \pm 4.6$ & $4.9 \pm 3.3$ & .46 \\
\hline \multicolumn{4}{|l|}{ Comorbidities (\%) } \\
\hline Hypertension & 50.0 & 68.0 & .16 \\
\hline Diabetes & 30.6 & 36.0 & .66 \\
\hline Congestive heart failure & 2.8 & 4.0 & .66 \\
\hline Insomnia & 5.6 & 20.0 & .09 \\
\hline Restless leg syndrome & 2.8 & 4.0 & .66 \\
\hline CPAP use, min/total days (\%) & $142.1 \pm 73.3$ & $347.9 \pm 92.7$ & $<.001$ \\
\hline CPAP use, days/total days & $63.5 \pm 26.8$ & $90.8 \pm 13.1$ & $<.001$ \\
\hline$>4 \mathrm{~h}$ use, days/CPAP use days & $41.7 \pm 19.4$ & $89.3 \pm 8.6$ & $<.001$ \\
\hline \multicolumn{4}{|c|}{$\begin{array}{l}\text { The values represent the mean } \pm \text { SD where applicable. The comorbidities indicate the percentage of subjects who were treated for each disease. } \\
\text { OSAS = obstructive sleep apnea syndrome } \\
\text { AHI = apnea hypopnea index }\end{array}$} \\
\hline
\end{tabular}

diary, and at the same time, downloaded the record of CPAP use from the CPAP device on the same day. The self-report of the CPAP start time in the sleep diary was utilized to adjust the time error of the CPAP device. Times during which the subjects reported that they had been awake lying in bed with CPAP were excluded from the analysis of body position.

\section{Definition of CPAP Removal During Sleep}

CPAP removal was defined as the discontinuation of CPAP therapy without the restart of CPAP therapy within $30 \mathrm{~min}$ after the subjects were lying in bed. We evaluated CPAP removal within $4 \mathrm{~h}$ from the start of body position recording with CPAP therapy, because the use of CPAP for $>4 \mathrm{~h}$ is usually regarded as good adherence.

For the subjects who had shown CPAP removal on both recording days, we utilized the record for the day on which CPAP removal occurred earlier from the start of body position recording when analyzing the concurrent removal of CPAP and a body position change.

\section{Analysis of Body Position During Sleep and Arterial Oxygen Desaturation Index (ODI)}

Each subject's body position (supine, left, right, prone, and upright) was recorded automatically every $1 \mathrm{~s}$. Body positions that did not last for $>1$ min were recorded as a continuation of the preceding body position. When referring to the events of body position changes, we did not include the changes from or to an upright position. An ODI of $3 \%$ or more was automatically analyzed after excluding periods including upright positions and the period when the subjects clearly reported that they were awake.

\section{Statistical Analysis}

The frequency of CPAP removal or simultaneous body position changes with CPAP removal events was evaluated using the chi-square test or Fisher's exact test. The number of body position changes and oxygen desaturations were analyzed among the poor adherers with/without CPAP removal and good adherers using the Mann-Whitney $U$ test for multiple comparisons with the $P$ values being corrected according to the Bonferroni method. The comparison of the ODI or the number of body position changes within 15 min before CPAP removal and that during other periods was performed using Wilcoxon matched-pairs (signed-rank) tests. The comparison of body position and number of body position changes before and after CPAP removal was also performed using Wilcoxon matched-pairs (signed-rank) tests. All of the statistical analyses were performed using the SPSS software program (version 21.0, SPSS, Chicago, Illinois). Statistical significance was defined as a $P$ value of $<0.05$. 


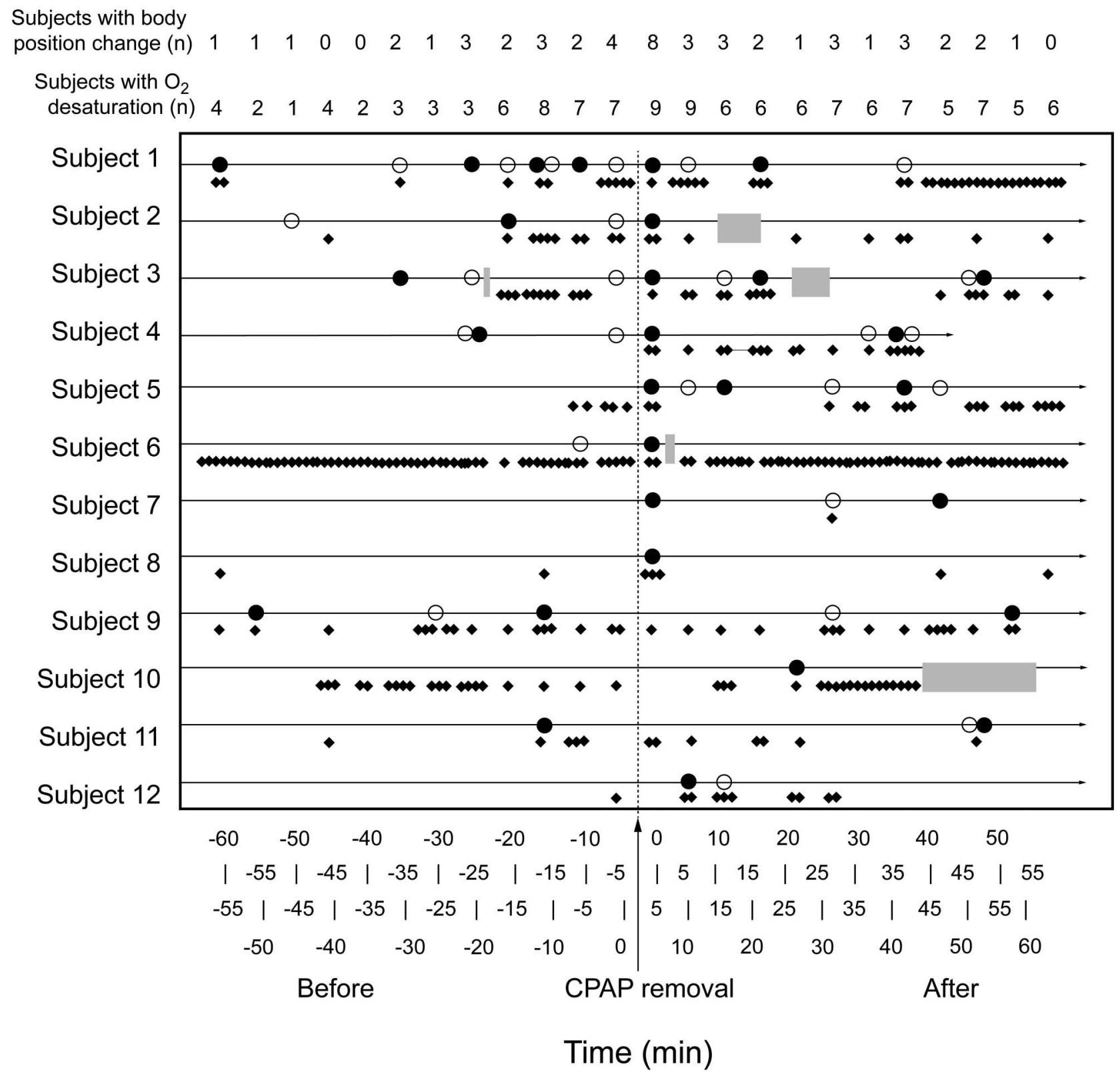

Fig. 1. Body position changes and oxygen desaturations around the time of CPAP removal. The horizontal axis shows the time course of the 12 subjects who experienced CPAP removal during the recording. The filled circles show body position changes from a supine position to non-supine positions, and open circles show body position changes from a non-supine position to a supine position. The diamonds show an oxygen desaturation of $\geq 3 \%$. The gray bars show the length of time that subjects were in an upright position.

\section{Results}

\section{Incidence of CPAP Removal During Sleep}

We evaluated body position during CPAP use in 36 of the 41 enrolled poor CPAP adherers and in 25 of the 28 enrolled good CPAP adherers. The remaining 5 poor CPAP adherers refused to undergo evaluation $(n=3)$ or completely withdrew from CPAP therapy before the recording of body position $(n=2)$. The 3 good CPAP adherers completely withdrew from CPAP therapy $(n=1)$ or became difficult to contact $(n=2)$. The characteristics of the evaluated CPAP users are shown in Table 1.

Twelve of the 36 poor CPAP adherers (33.3\%) exhibited subject removal of the CPAP device within $4 \mathrm{~h}$ from the start of body position recording, whereas none of the 25 good CPAP adherers $(0.0 \%)$ exhibited removal within $4 \mathrm{~h}$ from the start of body position recording $(P=.001$, Fisher's exact test). 


\section{Association of CPAP Removal and Body Position Changes}

Subsequently, we evaluated the association between CPAP removal and body position changes in 12 subjects who experienced CPAP removal during body position recording. The frequency of body position changes was remarkably high around CPAP removal (Fig. 1).

The number of body position changes increased just before CPAP removal in many cases. Seven of the 12 subjects (58.3\%) had changed their body position once or more within 15 min before CPAP removal. The frequency of body position changes was significantly higher within 15 min before CPAP removal than during other 15-min periods when CPAP was used $(P=.047$, Wilcoxon matched-pairs tests).

Subsequently, 8 of the 12 subjects $(66.7 \%)$ demonstrated a body position change within 5 min after CPAP removal. This percentage was significantly higher than that in the other 5-min periods of Figure 1, where 3 or fewer of the 12 subjects had made body position changes during each 5-min period ( $P=.041$, by using the chi-square test). Interestingly, all 8 body position changes within 5 min after CPAP removal occurred during a change from a supine position to a non-supine position, and 2 of the other 4 subjects had changed to a non-supine body position before CPAP removal, and thus 10 of the 12 subjects $(83.3 \%)$ were in a non-supine position $5 \mathrm{~min}$ after CPAP removal.

Although we could not evaluate awakening around the CPAP removal, none of the $12 \mathrm{CPAP}$ removal events were associated with an upright position observed before CPAP discontinuation (Fig. 1). In the sleep diaries of these 12 subjects, subject 6 reported that he had removed his CPAP device to go to the toilet. Subject 7 reported that she was awake for a short time at a time corresponding to a body position change subsequent to CPAP removal. Subject 3 reported that his sleep became poor at a time corresponding to $3 \mathrm{~min}$ after body position change, which occurred simultaneously with CPAP removal. The other subjects reported no events within 15 min before or after CPAP removal in their sleep diaries.

\section{Frequent Oxygen Desaturations Preceded CPAP Removal}

We also evaluated the association between oxygen desaturations and CPAP removal. Interestingly, a remarkably high number of subjects had shown oxygen desaturations of $3 \%$ or more before CPAP removal. The ODI within $15 \mathrm{~min}$ before CPAP removal was significantly higher than that during other 15 -min periods when CPAP was used ( $P=.041$, Wilcoxon matched-pairs tests). Interestingly, body position changes also occurred just be- fore or during these repetitive oxygen desaturations for many cases (subject 1, 2, 3, 6, 9, and 11) (Fig. 1).

\section{Number of Body Position Changes in the Subjects With CPAP Removal}

We hypothesized that some poor CPAP adherers did not remove the CPAP device because they remained in the supine position on the recoding days. Therefore, we evaluated the number of body position changes within $4 \mathrm{~h}$ from the start of body position recording. The number of body position changes during sleep was significantly lower in the 2-night data for the poor CPAP adherers who had not removed the CPAP device on either recording day compared with the 1-night or 2-night data for the 12 poor CPAP adherers who had removed CPAP therapy on one or both days $(P=.006)$ (Fig. 2). In particular, the number of the subjects who had not made any body position changes within the first $4 \mathrm{~h}$ was significantly higher in the poor CPAP adherers who had not removed the CPAP device (29.2\%), compared with the poor CPAP adherers who had removed the device $(0.0 \%)(P=.041$, Fisher's exact test). Furthermore, 5 of the 6 poor CPAP adherers who had removed the CPAP device on one night and had not removed it on the other night showed less frequent body position changes on the day without CPAP removal than on the day with CPAP removal, although the difference was not statistically significant $(P=.17$, Wilcoxon matched-pairs tests).

However, the number of body position changes within $4 \mathrm{~h}$ from the start of body position recording was not significantly different between poor CPAP adherers who had removed CPAP therapy and good CPAP adherers $(P=.12)$ (Fig. 2), or between total poor CPAP adherers and good CPAP adherers $(P=.57)$.

\section{Number of Oxygen Desaturations in the Subjects With CPAP Removal}

We hypothesized that suboptimal OSAS treatment could induce body position changes and CPAP removal. Therefore, we evaluated an ODI of 3\% or more within $4 \mathrm{~h}$ from the start of body position recording. As for the 12 subjects who had removed CPAP within $4 \mathrm{~h}$ from the start of body position recording, we evaluated an ODI of $3 \%$ or more from the start of body position recording to the time of CPAP removal. The ODI during sleep with CPAP was not significantly different among poor CPAP adherers who had removed CPAP therapy, poor CPAP adherers who had not removed CPAP therapy, and good CPAP adherers (Fig. 3). 


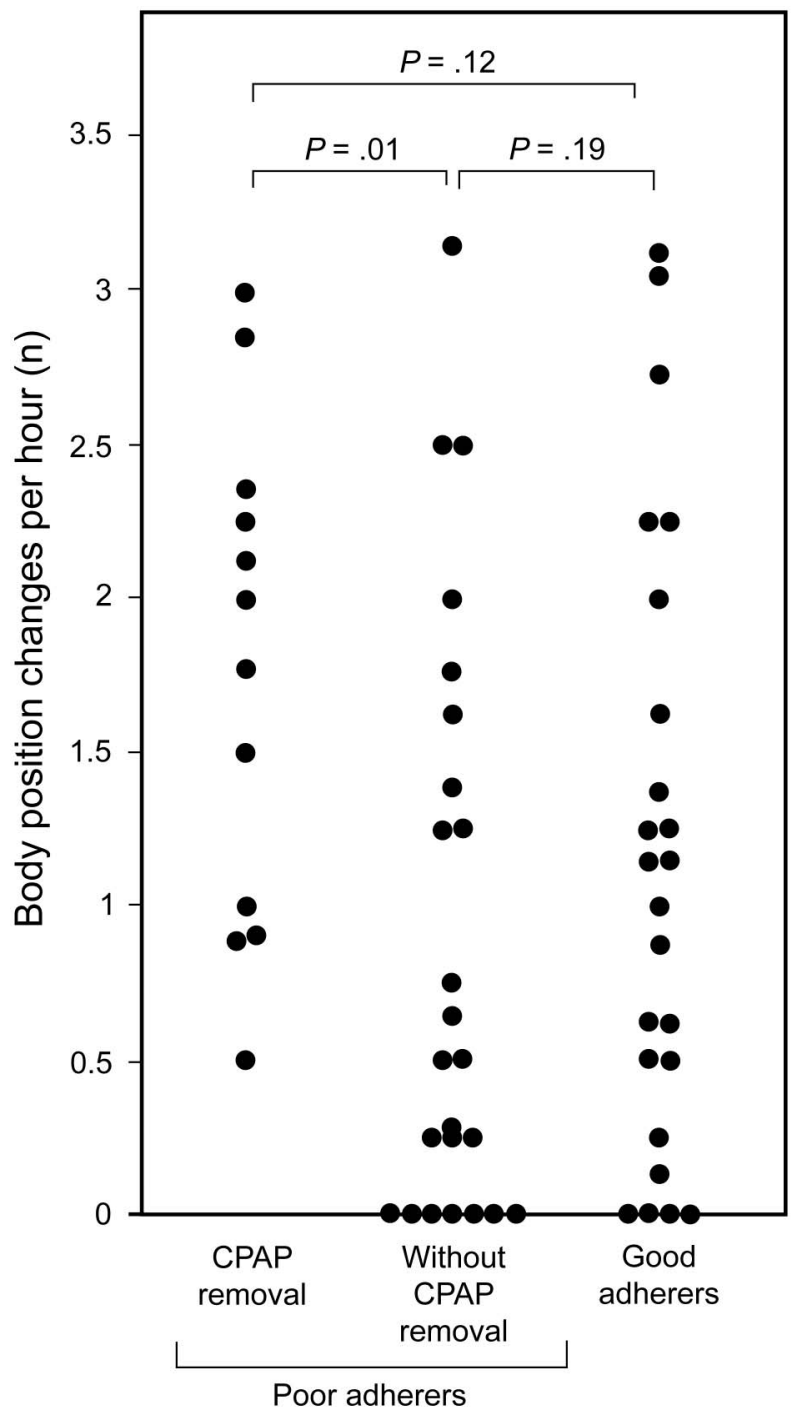

Fig. 2. Body position changes during sleep with CPAP therapy. The number of body position changes within $4 \mathrm{~h}$ from the start of body position recording was counted. The Mann-Whitney $U$ test for multiple comparisons was performed, with $P$ values corrected according to the Bonferroni method. These comparisons indicated that there was a significant difference between the poor CPAP adherers who had removed the CPAP device and the poor CPAP adherers who had not removed the CPAP device $(P=.006)$.

\section{Body Position and Body Position Changes After Subject Removal of CPAP Therapy}

For the 12 subjects who exhibited subject removal of the CPAP device, the percentage of non-supine body positions significantly increased during sleep after CPAP removal compared with that observed during sleep before CPAP removal $(P=.02)$ (Fig. 4A).

We also counted the number of body position changes during sleep before and after CPAP removal. The number of body position changes during sleep after CPAP removal

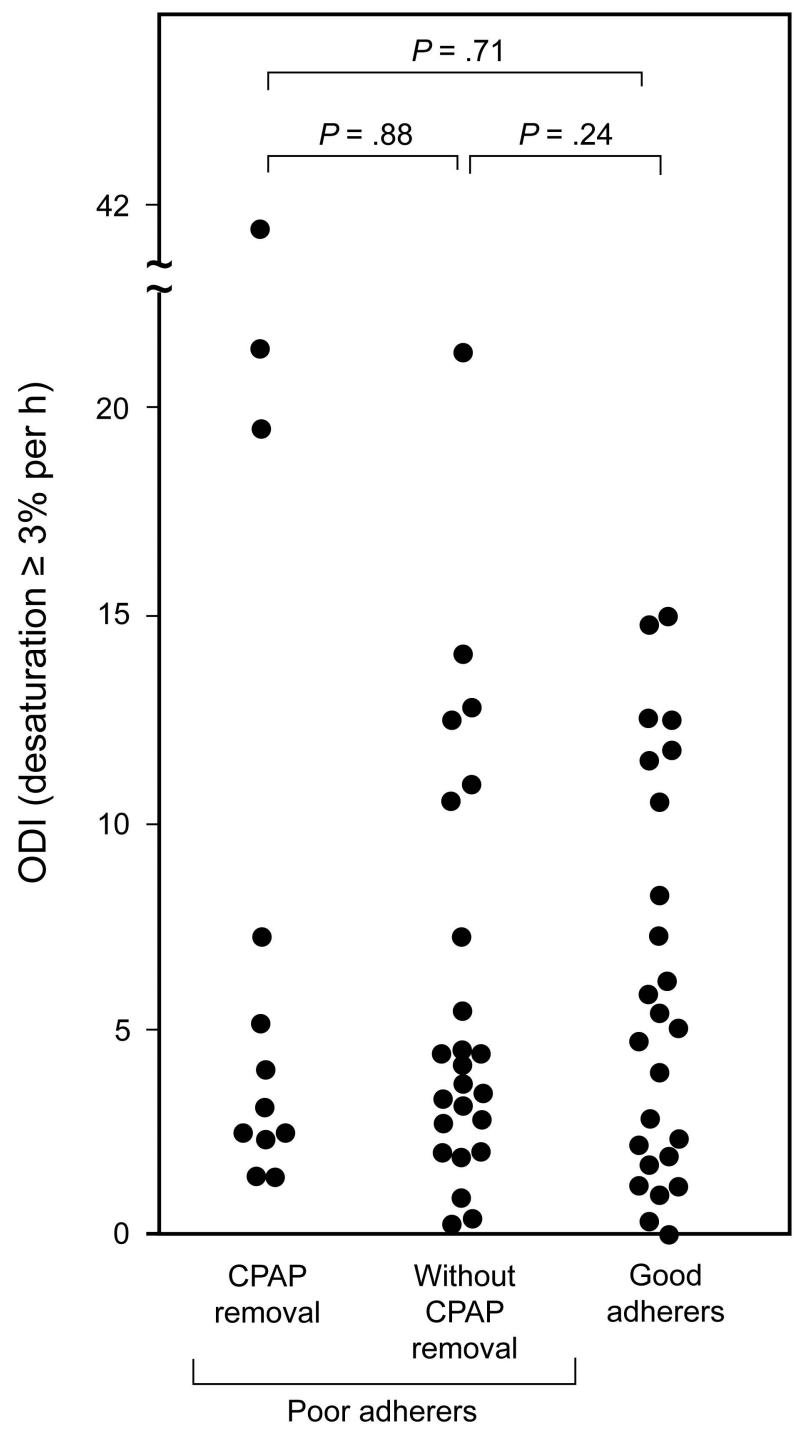

Fig. 3. An oxygen desaturation index (ODI) of $\geq 3 \%$ was counted within $4 \mathrm{~h}$ from the start of body position recording. For subjects who had removed CPAP within $4 \mathrm{~h}$ from the start of body position recording, we evaluated an ODI of $\geq 3 \%$ from the start of body position recording to the time of CPAP removal. The Mann-Whitney $U$ test for multiple comparisons was performed. These comparisons indicated that there were no significant differences among the poor CPAP adherers who had removed their CPAP device, the poor CPAP adherers who had not removed their CPAP device, and the good CPAP adherers.

was not significantly different from that observed during sleep before CPAP removal in the 12 subjects who exhibited subject removal of the CPAP device $(P=.12)$ (Fig. $4 B)$.

\section{Discussion}

In this study, we evaluated the associations among body position changes, oxygen desaturations, and the subject 

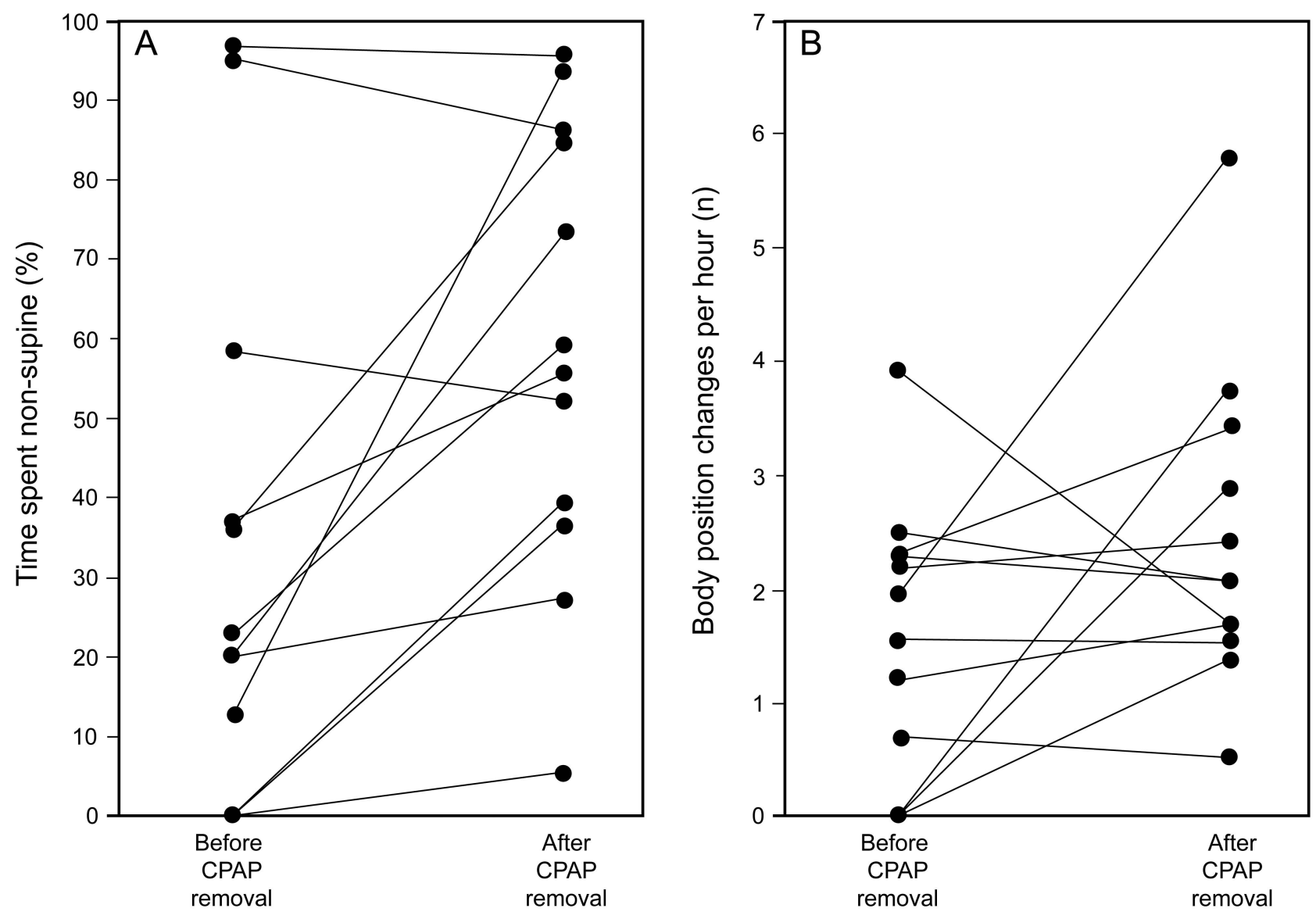

Fig. 4. Duration of non-supine positions and the number of body position changes before and after CPAP removal. A: In the 12 poor adherers who continued to remain in bed without CPAP after CPAP removal, the duration of time in non-supine body positions significantly increased after CPAP removal $(P=.02)$. B: In the 12 poor adherers who continued to remain in bed without CPAP after CPAP removal, the number of body position changes during sleep after CPAP removal was not significantly different from that observed during sleep before CPAP removal $(P=.24)$.

removal of the CPAP device during sleep. Although some CPAP users frequently remove the CPAP device during sleep, it has not been previously evaluated how CPAP removal occurs during sleep.

CPAP removal events occurred simultaneously with body position changes in many cases. Furthermore, we demonstrated that the subjects who removed the CPAP device during sleep tended to move into a non-supine position following CPAP removal. Unintentional effort to reduce the airway obstruction after CPAP removal would contribute to this association, because the duration of nonsupine sleep positions significantly increased during sleep after CPAP removal compared with that observed during sleep before CPAP removal.

Frequent oxygen desaturations just before CPAP removal events suggested that suboptimal OSAS treatment would induce body position changes and CPAP removal. An improved ability to set the pressure would help increase CPAP adherence for some poor CPAP adherers. At the same time, suboptimal OSAS treatment would not be the only reason for subjects removing their CPAP device during sleep because some good adherers also experienced frequent oxygen desaturations while using CPAP.

Interestingly, repetitive oxygen desaturations before CPAP removal were also preceded by body position changes in many cases. The CPAP mask would have been moved largely because of preceding body position changes, thus causing insufficient airway pressure before CPAP removal events. Although we could not clearly determine whether the discomfort at the time of body position changes caused the CPAP removal, the smaller number of body position changes in the poor CPAP adherers without CPAP removal suggests that maintaining the same body position may help to prevent CPAP removal.

At the same time, our observations showed that some subjects make unexpectedly frequent body position changes while using CPAP therapy, even among the good CPAP adherers. We speculate that patients adherent to CPAP therapy adapt to the discomfort of CPAP therapy at the time of body position changes. Better education in regard 
to mask fitting or the development of a CPAP mask or pillows that reduce the discomfort at the time of body position changes might also increase the CPAP adherence in some poor CPAP adherers.

There are some limitations associated with the present study. First, the number of subjects was relatively small. Second, we could not differentiate the CPAP removal with awakening from the CPAP removal without awakening because we did not measure electroencephalograms while recording body position. However, we speculate that many of the CPAP removals were not associated with longlasting awakening, because most of the CPAP removals were not associated with an upright position observed around the CPAP discontinuation. Furthermore, no subjects reported awakening before CPAP removal, and only 3 subjects reported awakening after CPAP removal in their sleep diaries.

Because intense monitoring systems would prevent natural sleeping and unintentional CPAP removal, we monitored only body positions and arterial oxygen saturations. We therefore cannot deny that identifying the time of CPAP removal may include some error, although the association among CPAP removal, body position changes, and oxygen desaturations would not be largely altered.

The topic of this study is completely novel, and the definition of CPAP removal during sleep, as well as standard procedure for the measurement of CPAP removal and a definition of good adherence have not been developed. The oxygen desaturations or body position changes occurring around CPAP removal had not been evaluated in any previous investigations. Our investigation could help clinicians to assess the reasons for CPAP removal during sleep and be the first step in creating an effective strategy for the prevention of CPAP removal during sleep.

\section{Conclusions}

Although we could not clarify the causal contribution of body position changes and oxygen desaturations to CPAP removal, our findings are the first to indicate a chronolog- ical association among CPAP removal, body position changes, and oxygen desaturations. Our findings also indicated that most good CPAP adherers make frequent body position changes while using CPAP therapy without removing the CPAP device, suggesting that patients can adapt to the discomfort of CPAP therapy at the time of body position changes.

\section{REFERENCES}

1. Bradley TD, Floras JS. Obstructive sleep apnoea and its cardiovascular consequences. Lancet 2009;373(9657):82-93.

2. Nannapaneni S, Ramar K, Surani S. Effect of obstructive sleep apnea on type 2 diabetes mellitus: a comprehensive literature review. World J Diabetes 2013;4(6):238-244.

3. Jenkinson C, Davies RJ, Mullins R, Stradling JR. Comparison of therapeutic and subtherapeutic nasal continuous positive airway pressure for obstructive sleep apnoea: a randomised prospective parallel trial. Lancet 1999;353(9170):2100-2105.

4. Campos-Rodriguez F, Peña-Griñan N, Reyes-Nuñez N, De la CruzMoron I, Perez-Ronchel J, De la Vega-Gallardo F, Fernandez-Palacin A. Mortality in obstructive sleep apnea-hypopnea patients treated with positive airway pressure. Chest 2005;128(2):624-633.

5. He J, Kryger MH, Zorick FJ, Conway W, Roth T. Mortality and apnea index in obstructive sleep apnea: experience in 385 male patients. Chest 1988;94(1):9-14.

6. Weaver TE, Grunstein RR. Adherence to continuous positive airway pressure therapy: the challenge to effective treatment. Proc Am Thorac Soc 2008;5(2):173-178.

7. Shapiro GK, Shapiro CM. Factors that influence CPAP adherence: an overview. Sleep Breath 2010;14(4):323-335.

8. Fox N, Hirsch-Allen AJ, Goodfellow E, Wenner J, Fleetham J, Ryan $\mathrm{CF}$, et al. The impact of a telemedicine monitoring system on positive airway pressure adherence in patients with obstructive sleep apnea: a randomized controlled trial. Sleep 2012;35(4):477-481.

9. Trupp RJ, Corwin EJ, Ahijevych KL, Nygren T. The impact of educational message framing on adherence to continuous positive airway pressure therapy. Behav Sleep Med 2011;9(1):38-52.

10. Falcone VA, Damiani MF, Quaranta VN, Capozzolo A, Resta O. Polysomnograph chart view by patients: a new educational strategy to improve CPAP adherence in sleep apnea therapy. Respir Care 2014;59(2):193-198.

11. Sawyer AM, Gooneratne NS, Marcus CL, Ofer D, Richards KC, Weaver TE. A systematic review of CPAP adherence across age groups: clinical and empiric insights for developing CPAP adherence interventions. Sleep Med Rev 2011;15(6):343-356.

This article is approved for Continuing Respiratory Care Education credit. For information and to obtain your CRCE

(free to AARC members) visit www.rcjournal.com

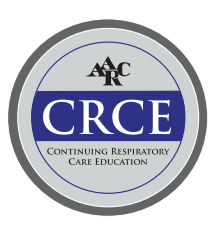

